\title{
Aprendizaje usando modelos BIM para motivar un conocimiento sostenible de los edificios e instalaciones de la Universidad de Oviedo.
}

\author{
Agustín Menéndez Díaz a, Celestino Ordoñez Galán 'b, Silverio García Cortes b, \\ Alfonso Lozano Luengas a , María Concepción Crespo Turrado c . \\ a Departamento de Construcción e Ingeniería de Fabricación, Universidad de Oviedo, \\ amenendez@uniovi.es, b Departamento de Explotación y Prospección de Minas, Universidad de \\ Oviedo $\mathrm{y}^{\mathrm{c}}$ Ingeniero de Mantenimiento de la Universidad de Oviedo. Vicerrectorado de Recursos \\ Materiales y Tecnológicos.
}

\begin{abstract}
The main goal that arises in this teaching innovation project, is to identify works and infrastructure of buildings and allow extract of the same parties that become problems of engineering for students. This search focuses on the works of reform, expansion, new installation or improvement of buildings of the University of Oviedo.

In many cases students only to see the classroom and the laboratory where he taught class, but have not stopped to think about the constructive elements, the functionality of the building they occupy, or its interaction with the environment. The buildings are our environment closer and it depends on our comfort, and therefore they are an ideal excuse to pose real problems, which help students to study and better understand the concepts of engineering.
\end{abstract}

The ultimate goal is to involve students and the University community in the knowledge of the buildings, their interaction with the environment, the sustainable maintenance of its facilities.

Keywords: CAD, BIM, Building Sustainability.

\begin{abstract}
Resumen
El objetivo principal que se plantea en este trabajo de innovación docente, es identificar obras e infraestructuras de edificios que se estén realizando, y que permitan extraer de las mismas partes que se conviertan en problemas dosificados para los alumnos de ingeniería. Para ello se plantea poner el foco de esta búsqueda en las obras de reforma, ampliación, nueva instalación o de mejora de los edificios de la propia Universidad de Oviedo.

En muchos casos los alumnos solo ven el aula o el laboratorio donde imparten clase, pero no se han parado a pensar los elementos constructivos, la funcionalidad del edificio que ocupan, o su interacción con el entorno. Los edificios son nuestro medio ambiente más próximo y de ello depende nuestra comodidad y nuestro confort, y por lo tanto son una excusa ideal para plantear problemas reales, que ayuden a los alumnos a estudiar y
\end{abstract}


comprender mejor, los conceptos propios de cada rama tecnológica de la ingeniería.

El fin último es involucrar a los alumnos y a la comunidad universitaria en el conocimiento de los edificios, su interacción con el medio ambiente, el mantenimiento sostenible de sus instalaciones

Palabras clave: CAD, BIM, Sostenibilidad de Edificios.

\section{Introducción}

El planteamiento en este trabajo es presentar un proyecto innovación docente cuyo fin es identificar problemas reales en los edificios e infraestructuras universitarias, que puedan ser presentados a los alumnos como problemas aplicados de su futuro trabajo profesional. Realizando visitas a las obras que se están ejecutando para mejorar esos edificios, se ha buscado que los alumnos conozcan los edificios de su Universidad, analicen la necesidad de las obras ejecutadas, y desde un espíritu crítico sean capaces de identificar soluciones sostenibles a los problemas planteados. El elemento conductor que se plantea en este proyecto de innovación docente, son las obras de reforma, ampliación, y mejora de instalaciones en los edificios de la propia Universidad de Oviedo.

Cuando en las instalaciones de la Universidad se hacen obras estos espacios se clausuran y muchas veces no puede accederse a ellos (“Cerrado por Obras”). El planteamiento de este trabajo, es abrir esos espacios de los edificios para que los alumnos conozcan las obras (“Abierto por Obras”) y presentarles directamente los problemas específicos de ingeniería que se están resolviendo en ese momento.

Habitualmente los alumnos solo ven el aula o el laboratorio donde imparten clase, pero no se han parado a pensar en los elementos constructivos y la funcionalidad del edificio que ocupan, en su interacción con el entorno y en el coste tanto medio ambiental como económico derivado de su explotación, así como en la propia relación alumno-edificio, grado de confort, comodidad etc. Estas obras pueden ser una excusa ideal para plantear problemas reales, que ayuden a los alumnos a comprender mejor los conceptos propios de cada rama tecnológica que estén estudiando.

Trabajos similares han sido desarrollado en proyectos educativos como los descritos en [Liu and Hatipkarasulu, 2014], [McGough et al., 2013] y [Pikas et al., 2017]. En la mayor parte de los casos, la metodología educativa se articula sobre los sistemas de modelado de edificios BIM (Building Information Modeling) [Han and Golparvar-Fard, 2017], integrando elementos más específicos como los de sostenibilidad a partir de los problemas energéticos [Choi, 2016].

Por otro lado, este proyecto de innovación se ha realizado teniendo en cuenta alumnos de tres asignaturas que son: Expresión Gráfica (31 alumnos) del Grado de Ingeniería Química, Fotogrametría II (10 alumnos) del Grado en Ingeniería en Geomática y Topografía, y Construcción (27 alumnos) del Grado en Ingeniería Forestal. Han participado en el mismo, profesores de tres áreas de conocimiento (Expresión Gráfica, Explotación de Minas,

(cc) EY-NC-ND 2018, Universitat Politècnica de València

Congreso IN-RED (2018) 
Construcción), así como la ingeniera de mantenimiento del Vicerrectorado de Recursos Materiales y Tecnológicos de la Universidad de Oviedo.

\section{Objetivos y herramientas previas basadas en BIM.}

El objetivo principal que se plantea en este proyecto de innovación, es identificar problemas reales de las infraestructuras universitarias, analizar ejemplos específicos y buscar soluciones desde una perspectiva de desarrollo sostenible, que puedan ser estudiados por los alumnos insitu, involucrándolos desde sus áreas de conocimiento.

Para desarrollar este objetivo general se han cubierto una serie de objetivos específicos:

- Identificar proyectos singulares de obras en la universidad, y plantearlos como ejercicios de curso para los alumnos.

- Desarrollar modelos virtuales que simulen el estado presente o futuro que va a tener un edifico, antes y después de una obra nueva o una obra de mantenimiento. Se utilizarán técnicas de modelado virtual, como el uso de sistemas de registro digitales usando scanner 3D y fotogrametría, así como programas de diseño CAD (Computer Aided Design) y técnicas BIM (Building Information Modeling).

- Involucrar a los alumnos y a la comunidad universitaria en el conocimiento de los edificios, su interacción con el medio ambiente, el mantenimiento de sus instalaciones y el uso del mismo desde una visión sostenible.

- Plantear proyectos fin de carrera que den soluciones similares o alternativas a obras proyectadas o en ejecución dentro de la universidad.

Para realizar dichos objetivos, fue fundamental usar y desarrollar una serie de herramientas que nos permitieron traducir los problemas técnicos de los edificios en un BIM. El resultado de dichas herramientas fueron una serie de maquetas virtuales que pudieron ser manipuladas y consultadas por los alumnos, para comprender toda la complejidad de los elementos estructurales y de las instalaciones de cada edificio.

Para cada edificio se partió de los planos en papel y de los datos espaciales suministrados por un escaner 3d (RIEGL LMS-Z390i). Se procedió a la digitalización de los edificios y de sus instalaciones obteniendo una nube de puntos 3D lo más fidedigna posible (ver Fig 1). Para ello se usó el programa RISCAN PRO que trabaja sobre datos del escáner georefenciando y filtrando los mismos. A continuación se clasificaron usando el programa AutoDesk RECAP.

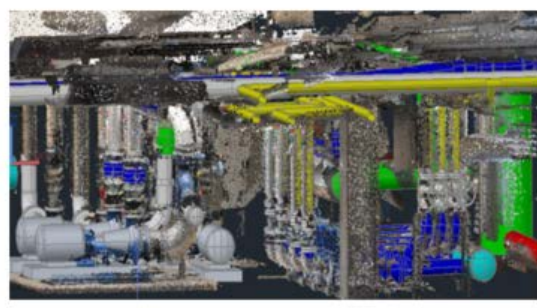

Láser escáner terrestre RIEGL LMS-Z390i

- Precisión $6 \mathrm{~mm}$

- Área de toma: $80^{\circ}$ vertical y $360^{\circ}$ horizontal

Nube de puntos

RISCANPRO

RECAP

(c) $)$ EY-NC-ND 2018, Universitat Politècnica de València

Congreso In-Red (2018) 
Fig. 1 Herramientas usadas para generar, filtrar y clasificar las nubes de puntos escaneadas.

Posteriormente se identificaron las redes de tuberías con AutoDesk PLANT y paralelamente con el programa REVIT, se hizo lo mismo con los elementos estructurales (paredes, forjados, cubiertas, paramentos, etc). Finalmente con las herramientas de NAVISWORK 3D (ver Fig 2) se montaron las maquetas virtuales de los edificios, sobre las que se sustenta todo el proyecto de innovación que se describe a continuación.

\section{AUTOCAD PLANT}
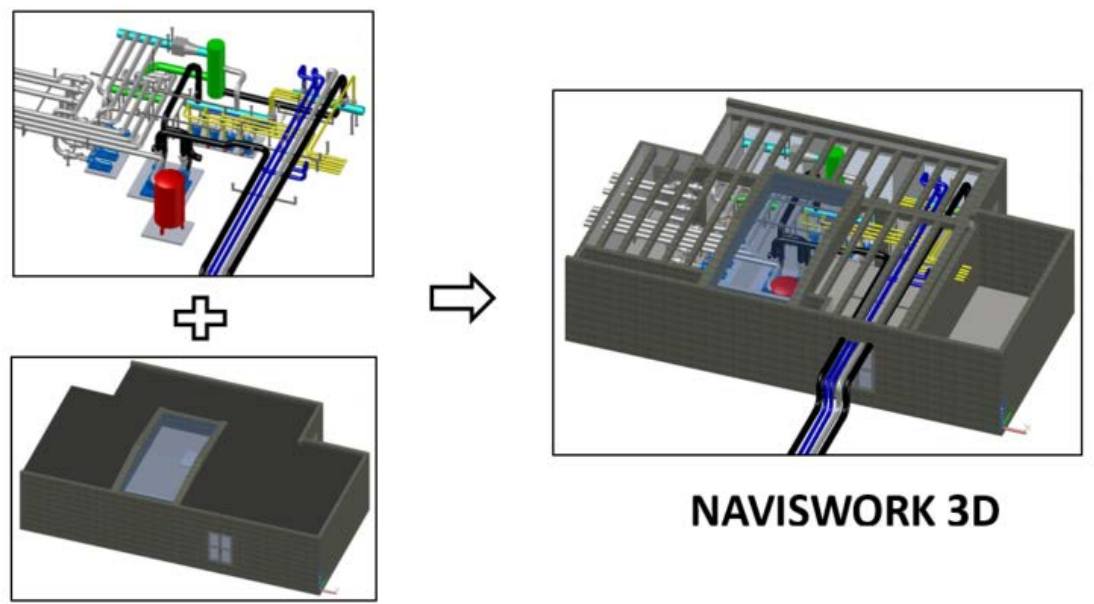

NAVISWORK 3D

\section{REVIT}

Fig. 2 Herramientas usadas para generar los modelos BIM de las instalaciones y edificios.

\section{Metodología desarrollada.}

En el desarrollo del proyecto se han identificado cuatro proyectos singulares de obras en la universidad que son:

- Sistema de ventilación de formaldehido en la Facultad de Medicina.

- Sistema de calefacción de la Facultad de Química.

- Sistema de calefacción con la sala de calderas y de bombeo de la EPM (Escuela Politécnica de Mieres).

- La auditoría de la eficiencia energética en edificios y el desarrollo de una Red Calor en el Campus de Llamaquique (Se tomó como referencia para los PFG y PFM).

La idea fue presentar ordenadamente cada uno de estos proyectos a los alumnos, aprovechando las obras que se estaban realizando en ese momento. Sin embargo, el primer día de clase de Expresión Gráfica de Ingeniería Química, se produjo una avería en la calefacción de la Facultad de Químicas que afectó la actividad docente del centro y que tardó en ser resulta dos días por el Servicio de Mantenimiento de la Universidad de Oviedo. La noticia apareció en prensa (ver Fig 3-a) y fue el pretexto ideal para explicar este problema en clase. 
El paradigma de “abierto por obras”, se transformó en “abierto por avería”. Se les explicó a los alumnos lo que había pasado, porque había sido necesario trasladar parte de las clases a otro lugar, porque habían sido afectadas unas partes del edificio y otras no. Todo esto se documentó mediante planos y fotografía para explicar la avería.

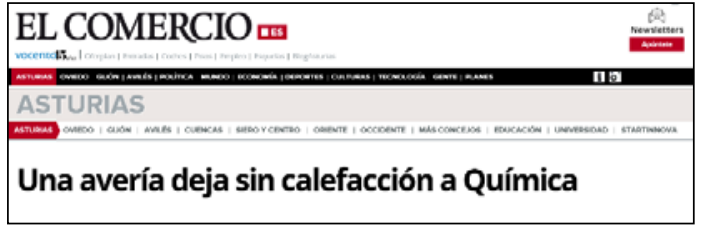

a)

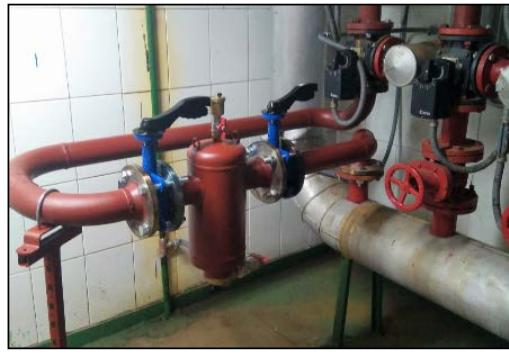

b)

Fig. 3 Noticia que apareció el primer día de clase en la Fac. de Químicas y reparación realizada.

Posteriormente se hizo una visita a las instalaciones de la sala de caldera de la Facultad de Químicas, explicando el funcionamiento de la sala y la reparación realizada (Fig 3-b). Esta consistió básicamente en el montaje de una nueva bomba de recirculación y de un nuevo filtro de agua. En las clases teóricas se explicó el plano de sección de la bomba, y se hizo especial referencia a la necesidad de instalar los filtros adecuadas en la red de tuberías.

Con este caso, queremos indicar la filosofía básica de este proyecto de innovación docente, que es aprovechar cualquier obra o reparación que se produzca en los edificios de la universidad, para que los alumnos puedan aprender de ellas. En ese mismo curso, se hizo un trabajo de tuberías, más detallado que ampliaba el conocimiento que los alumnos deben de tener sobre una sala de calderas, explicando el resto de elementos básicos (tipos de calderas, compensadores hidráulicos, colectores, válvulas de recirculación, válvulas de mezclado, vaso de expansión, etc).

El resto de visitas se ajustó a una planificación similar. Todas las actividades se iniciaban con una charla expositiva de unos 45 min y a continuación una visita a los edificios y a sus instalaciones de otros 30 min. En las siguientes Figs $(4,5,6,7)$ se indican varias salidas, así como las maquetas virtuales y planos utilizados para documentar cada instalación.

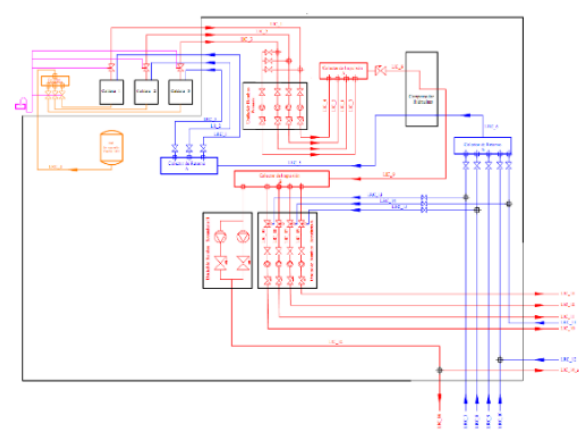

a)

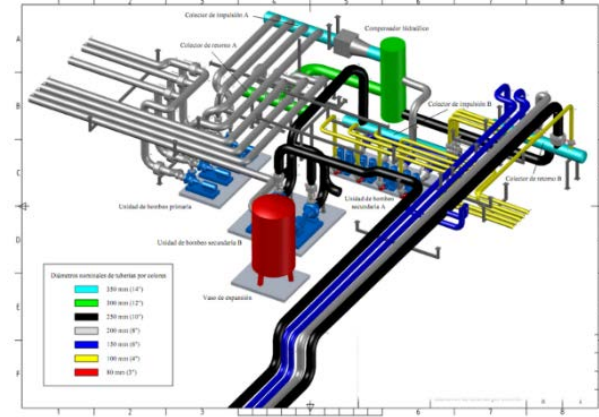

b)

Fig 4. Esquema y maqueta virtual usando BIM de una sala de calderas y de bombeo de la EPM. 


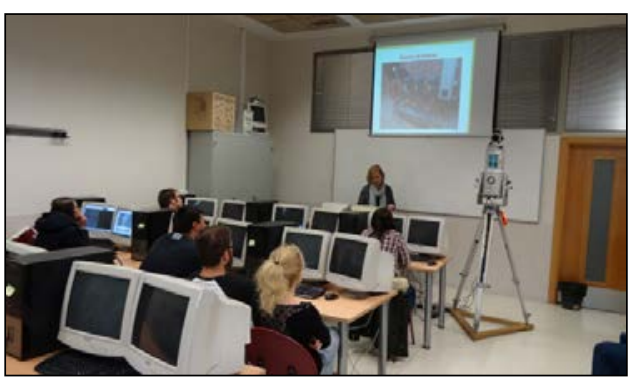

a)

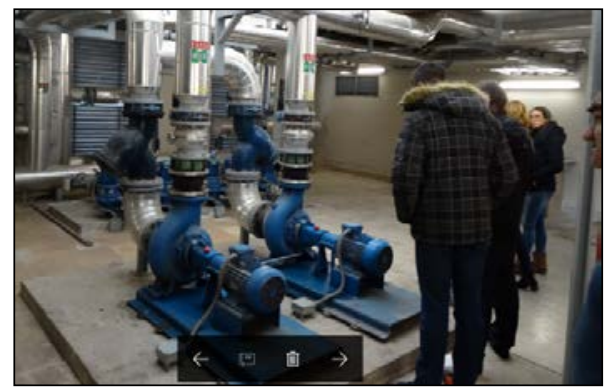

b)

Fig 5. Charla a Ing. Geomática con el escaner a) y visita posterior a las instalaciones b).

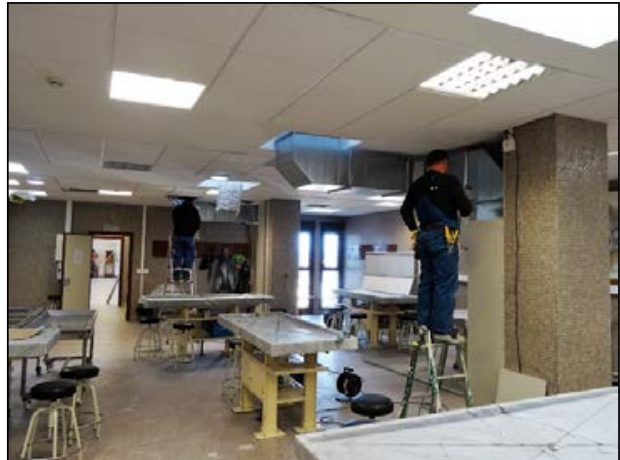

a)

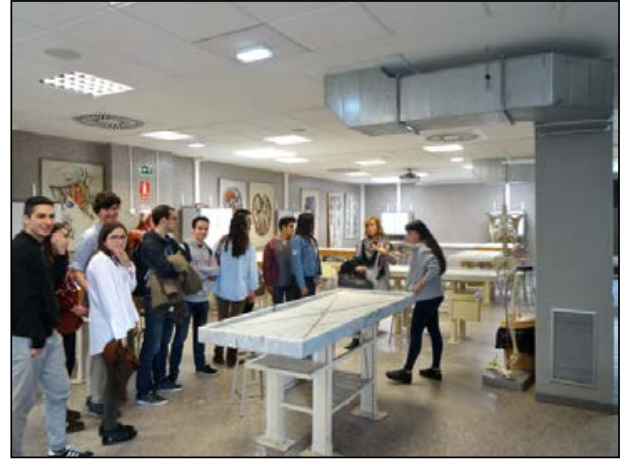

b)

Fig 6. Visita a la sala de disecciones de la Fac. de Medicina de los alumnos de Ing. Química.

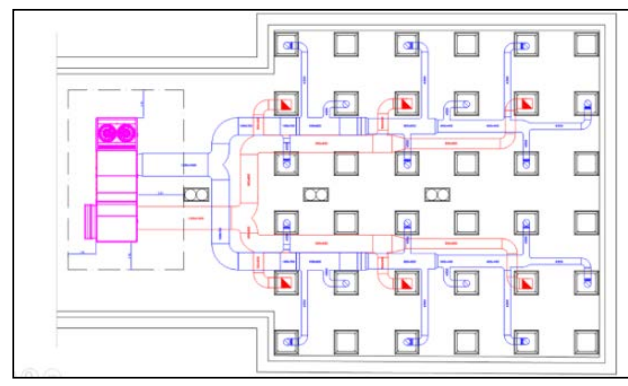

a)

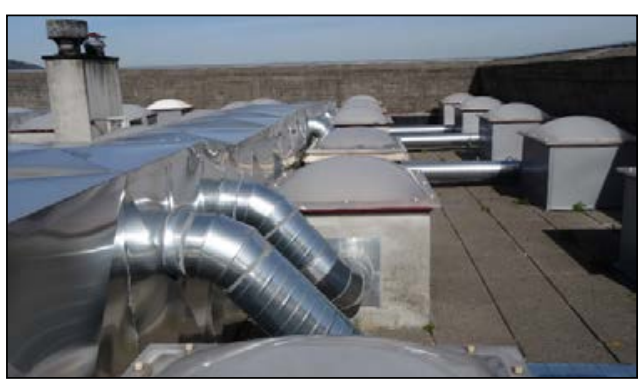

b)

Fig 7. Disposición del sistema de ventilación de formaldehido en la azotea de la Fac. de Medicina.

Todo este trabajo se organizó en seis fases:

- Fase 1: Identificación de los problemas en cada edificio.

- Fase 2: Realización de modelos virtuales usando técnicas CAD y BIM.

- Fase 3: Elaboración de presentaciones usando diferentes soportes (videos, reportajes fotográficos, presentaciones) para explicar cada obra.

(cc) EY-NC-ND 2018, Universitat Politècnica de València

Congreso IN-RED (2018) 
- Fase 4: Realización de charlas (45 minutos) y las visitas a las obras directamente a continuación (30 minutos).

- Fase 5: Discusión en clase con los profesores de otras titulaciones de las instalaciones visitadas.

- Fase 6: Realización de trabajos de curso relacionados con estas visitas.

La Fase 1 y Fase 2 se realizaron antes de iniciar el segundo cuatrimestre. Se tomó como referencia los edificios en los que cada alumno recibe sus clases. El objetivo es que el alumno de cada edificio, conociese aspectos de los mismo que desconocía. La Fase 3 se realizó durante el mes de enero, una vez que ya había finalizado la obra de la sala de disecciones de la Facultad de Medicina. Para esas fechas también se compiló toda la información disponible en formato digital de las salas de calderas y de bombeo que se visitaron. La Fase 4 se concretó en tres charlas y dos visitas en Ingeniería Química, mientras que en las asignaturas de la EPM se hicieron dos charlas y se realizaron dos visitas a la sala de calderas y a la sala de bombeo del sistema ACS (Agua Caliente Sanitaria) del edificio. La Fase 5 se realizó solamente en Ingeniería Química en la que todos los alumnos debían presentar una colección de 10 planos en DWG (AutoCAD) presentando varias instalaciones, entre ellas, el prediseño de la sala de disecciones de la Facultad de Medicina.

En la Fase 1 participamos todos los profesores del proyecto de innovación docente, aportando cada uno nuestro punto de vista, y desarrollando los problemas fundamentales en las obras a realizar en los edificios. Así los profesores de topografía se centraron más en el escaneo mediante de los espacios donde realizar las obras, el profesor de Expresión Gráfica en la recopilación de todos los planos e información gráfica necesaria para explicar la obra, y el profesor de Construcción en desglosar cada parte de la obra a ejecutar, analizando los elementos estructuras prexistentes y como estos iban a ser modificados.

La elaboración de todo el material gráfico y de maquetas virtuales corrió a cargo fundamentalmente de Agustin Menendez y Silverio García (Fases 2 y 3). Y en las charlas más específicas de cada asignatura, participó el profesor cada asignatura (Agustin Menéndez, Silverio Castro y Alfonso Lozano) así como la responsable de mantenimiento de la Universidad, Concha Crespo (Fase 4). Por ultimo Celestino Ordoñez participó en la Fase 5 y fue el encargado de realizar las encuestas y la revisión de los trabajos presentados.

Para completar las temáticas propuestas en el proyecto, durante su desarrollo se ha iniciado la realización de dos proyectos fin de grado y de un proyecto fin de master. Dichos proyectos fueron dirigidos por Alfonso Lozano y Agustin Menendez, y también colaboró con el profesor Francisco Javier Iglesias que estaba desarrollando un proyecto sobre eficiencia energética en la Escuela de Minas de Oviedo.

El desarrollo de las charlas y la realización de las modelizaciones y maquetas virtuales, se realizó en tres asignaturas que son: Expresión Gráfica (31 alumnos) del Grado de Ingeniería Química, Fotogrametría II (10 alumnos) del Grado en Ingeniería en Geomática y Topografía, y Construcción (27 alumnos) del Grado en Ingeniería Forestal. Han participado en el mismo, profesores de tres áreas de conocimiento (Expresión Gráfica, Explotación de 
Minas, Construcción), así como la ingeniera de mantenimiento del Vicerrectorado de Recursos Materiales y Tecnológicos de la Universidad de Oviedo.

Respecto a los recursos empleados, se ha utilizado el material didáctico de cada una de las asignaturas, ya disponible en el campus virtual como hasta ahora, pero complementada con planos y modelos virtuales que permitan a los alumnos comprender cada obra o actuación.

Para ello, se facilitaron planos de obras que pudieron ser consultados por los alumnos sobre los elementos estructurales diseñados, así como los proyectos de ejecución e inventarios fotográficos de los que disponía. Esta parte fue coordinada en este proyecto docente por la ingeniera de instalaciones de la Universidad de Oviedo (Concha Crespo Turrado). Toda esta documentación se ha obtenido de obras similares a las ejecutadas, así como de información facilitada por el Vicerrectorado de Recursos Materiales y Tecnológicos.

La elaboración de las maquetas virtuales se ha realizado usando los programas de software con los que se imparten la clase de CAD y BIM en los distintos centros, utilizando los equipos del Aula de Fotogrametría de Mieres, así como los medios informáticos de la sala de RPAS (Remotely Piloted Aircraft Systems) de la EPM.

\section{Resultados alcanzados y evaluación.}

Son resultados directos que avalan el desarrollo del proyecto, los siguientes:

- La presentación a los alumnos de obras y trabajos reales que se desarrollan en los edificios de la universidad.

- La elaboración de planos usado técnicas gráficas y geomáticas para hacer modelos virtuales de las obras realizadas.

- La elaboración de inventarios fotográficos de las obras que permiten estudiar las mismas y ver su evolución en el tiempo.

- El desarrollo de dos PFG y un PFM ligados a los edificios de la propia Universidad de Oviedo.

Para estimar dichos resultados se realizó la estimación de 4 indicadores que fueron:

1. Número de aprobados en cada asignatura.

2. Porcentaje de alumnos que participen en las actividades, realizando además una encuesta de autoevaluación.

3. Número de trabajos o modelos virtuales elaborados por los estudiantes, relacionados con edificios de la Universidad.

4. Número de TFG y TFM iniciados en la temática de Sostenibilidad de Edificios.

(cc) EY-NC-ND 2018, Universitat Politècnica de València

Congreso IN-RED (2018) 
Los resultados de dichos indicadores fueron los siguientes (ver Tabla 1):

Tabla 1. Criterio de Evaluación: valoración obtenida

\begin{tabular}{|c|l|l|l|}
\hline $\mathbf{N}^{\mathbf{0}}$ & \multicolumn{1}{|c|}{ Indicador } & \multicolumn{1}{|c|}{ Justificación } & \multicolumn{1}{|c|}{ Resultados Obtenidos } \\
\hline 1 & Número de aprobados en cada asignatura & $\begin{array}{l}\text { Notas una vez realizados todos los } \\
\text { exámenes ordinarios y extraordinarios }\end{array}$ & $\begin{array}{l}\text { Aprobados }=70,59 \% \\
70 \%-100 \%-\text { Bueno }\end{array}$ \\
\hline 2 & $\begin{array}{l}\text { Porcentaje de alumnos que participen en las } \\
\text { actividades, realizando además una } \\
\text { encuesta de autoevaluación. }\end{array}$ & $\begin{array}{l}\text { Se controlará la asistencia de los } \\
\text { alumnos a las obras a obras y labores } \\
\text { de mantenimiento en los edificios, } \\
\text { evaluando con una encuesta su } \\
\text { participación. }\end{array}$ & $\begin{array}{l}\text { Alumnos participantes }=51 \\
\text { Valor obtenido }=75 \% \\
70 \%-100 \%-\text { Bueno }\end{array}$ \\
\hline 3 & $\begin{array}{l}\text { Número de trabajos o modelos virtuales } \\
\text { elaborados por los estudiantes, relacionados } \\
\text { con edificios de la Universidad }\end{array}$ & $\begin{array}{l}\text { Se evaluará el ratio: trabajos } \\
\text { presentados/número de alumnos } \\
\text { totales }\end{array}$ & $\begin{array}{l}\text { Trabajos presentados }=26 \\
\text { Ratio }=26 / 68=0.38 \\
0.3<\text { Ratio }<0.8 \text { Aceptable }\end{array}$ \\
\hline 4 & $\begin{array}{l}\text { Número de TFG y TFM iniciados en la } \\
\text { temática de Sostenibilidad de Edificios. }\end{array}$ & $\begin{array}{l}\text { Se aporta carta de aceptación de } \\
\text { proyectos PFC y TFM en realización. }\end{array}$ & 3 proyectos - Aceptable \\
\hline
\end{tabular}

El criterio indicador $\mathrm{N}^{\circ} 1$ viene justificado en la Tabla 2 donde se indica el número de aprobados en cada asignatura en función del número de alumnos matriculados.

Tabla 2. Criterio de Evaluación: número de aprobados.

\begin{tabular}{|l|l|c|c|c|}
\hline \multicolumn{1}{|c|}{ Asignatura } & \multicolumn{1}{|c|}{ Titulación } & $\begin{array}{c}\text { Número } \\
\text { total } \\
\text { de alumnos }\end{array}$ & $\begin{array}{c}\text { Número } \\
\text { de } \\
\text { aprobados }\end{array}$ & $\begin{array}{c}\text { \% } \\
\text { Aprobados }\end{array}$ \\
\hline Expresión Gráfica & Grado en Ingeniería Química & $\mathbf{3 1}$ & $\mathbf{2 3}$ & $74,19 \%$ \\
\hline Fotogrametría II & Grado en Ingeniería en Geomática y Topografía & $\mathbf{1 0}$ & $\mathbf{9}$ & $90,00 \%$ \\
\hline Construcción & Grado en Ingeniería Forestal & $\mathbf{2 7}$ & $\mathbf{1 6}$ & $59,26 \%$ \\
\hline Total & Todas & $\mathbf{6 8}$ & $\mathbf{4 8}$ & $\mathbf{7 0 , 5 9 \%}$ \\
\hline
\end{tabular}

Respecto al criterio indicador $\mathrm{N}^{\circ} 2$ indicar que el número de alumnos que participo fue de 30 en Expresión Gráfica, unos 4 en Fotogrametría II (había 4 alumnos polacos de Erasmus que al no impartirse las clases en inglés no asistieron a las actividades de forma regular), y de los alumnos de Construcción participaron unos 17 alumnos. El número de alumnos que participó asiduamente fue de 51 personas (el $75 \%$ de los matriculados).

Como complemento a estos valores obtenidos resumimos también los resultados de la encuesta realizada por los alumnos. La encuesta se articuló en 8 preguntas que se recogen en la Tabla 3. Cada pregunta se ha contestado indicando 5 niveles de satisfacción: 1mínimo, 2, 3, 4, 5-máximo. La encuesta fue anónima y voluntaria, recibiendo se un total de 18 respuestas. La participación en la encuesta fue del 35 \% de los alumnos asistentes, debido en parte a que la encuesta se realizó a finales del segundo cuatrimestre, cuando los alumnos ya están inmersos en el periodo de exámenes. Se aprecia que hubo una mayor participación en aquellos centros en los que se hicieron más actividades, como es el caso de Ingeniería Química. En todo caso, esta encuesta arroja una valoración muy positiva en casi todas las preguntas realizadas. 
A modo de resumen, si consideramos la Pregunta 7 "Indícanos tu grado de interés en asistir a actividades similares en próximos cursos”, las contestaciones fueron las siguientes:

- 1 nivel de satisfacción mínimo (0 \%).

- 2 nivel de satisfacción bajo (0 \%).

- 3 nivel de satisfacción medio (11\%).

- 4 nivel de satisfacción alto (39\%).

- 5 nivel de satisfacción máximo. (50 \%).

Por otro lado, el 89 \% de los encuestados repetiría en años sucesivos claramente una actividad similar a la implementada en este proyecto de innovación docente.

Tabla 3. Criterio de Evaluación: número de aprobados

\begin{tabular}{|c|c|}
\hline $\begin{array}{c}\text { ID } \\
\text { PREGUNTA }\end{array}$ & TEXTO DE LA PREGUNTA \\
\hline $\mathbf{0 .}$ & $\begin{array}{l}\text { Indica la titulación en la que estas matriculado. } \\
\text { 1. Grado en Ingeniería Química } \\
\text { 2. Grado en Ingeniería Forestal } \\
\text { 3. Grado en Ingeniería en Geomática y Topografía }\end{array}$ \\
\hline 1. & $\begin{array}{l}\text { Consideras que las charlas dadas sobre los edificios de la Universidad han sido positivas. } \\
\text { Valora de } 1 \text { a } 5 \text { según tu grado de satisfacción ( } 1 \text { - mínimo, } 5 \text { - máximo) }\end{array}$ \\
\hline 2. & $\begin{array}{l}\text { Consideras que las visitas a instalaciones de edificios realizadas han sido didácticas. } \\
\text { Valora de } 1 \text { a } 5 \text { según tu grado de satisfacción ( } 1 \text { - mínimo, } 5 \text { - máximo) }\end{array}$ \\
\hline 3. & $\begin{array}{l}\text { Consideras adecuada la participación en las charlas de técnicos de otras especialidades. } \\
\text { Valora de } 1 \text { a } 5 \text { según tu grado de satisfacción ( } 1 \text { - mínimo, } 5 \text { - máximo) }\end{array}$ \\
\hline 4. & $\begin{array}{l}\text { Consideras que las actividades desarrolladas son más prácticas que teóricas. } \\
\text { Valora de } 1 \text { a } 5 \text { según tu grado de satisfacción ( } 1 \text { - mínimo, } 5 \text { - máximo) }\end{array}$ \\
\hline 5. & $\begin{array}{l}\text { Las actividades desarrolladas te han permitido conocer mejor los edificios de la Universidad. } \\
\text { Valora de } 1 \text { a } 5 \text { según tu grado de satisfacción ( } 1 \text { - mínimo, } 5 \text { - máximo) }\end{array}$ \\
\hline 6. & $\begin{array}{l}\text { De las actividades desarrolladas has podido encontrar temas en los que podrías desarrollar tu proyecto } \\
\text { fin de carrera al final de tus estudios. } \\
\text { Valora de } 1 \text { a } 5 \text { según tu grado de satisfacción ( } 1 \text { - mínimo, } 5 \text { - máximo) }\end{array}$ \\
\hline 7. & $\begin{array}{l}\text { Indícanos tu grado de interés en asistir a actividades similares en próximos cursos. } \\
\text { Valora de } 1 \text { a } 5 \text { según tu grado de satisfacción ( } 1 \text { - mínimo, } 5 \text { - máximo) }\end{array}$ \\
\hline 8. & $\begin{array}{l}\text { Indique otros aspectos técnicos que te gustaría conocer de los edificios de la Universidad. } \\
\text { Valora de } 1 \text { a } 5 \text { según tu grado de satisfacción ( } 1 \text { - mínimo, } 5 \text { - máximo) }\end{array}$ \\
\hline
\end{tabular}

Por último, los tres proyectos desarrollados para justificar el criterio indicador $\mathrm{N}^{\circ} 4$ son:

- Título del Proyecto Fin de Grado: "Estudio de la eficiencia energética de un edificio de la Universidad de Oviedo situado en el Campus de Llamaquique”. Titulación Ingeniería Mecánica. Director del Proyecto: Alfonso Lozano Martínez Luengas.

- Título del Proyecto Fin de Master: "Estudio viabilidad técnica y económica de una instalación solar térmica para satisfacer parte de la demanda de calefacción en la Escuela de Ingeniería de Minas, Energía y Materiales de Oviedo”. Titulación Master en Ingeniería de Minas. Director del Proyecto: Francisco Javier Iglesias Rodríguez.

- Título del Proyecto Fin de Grado: "Desarrollo de una red de calor en el campus de Llamaquique”. Titulación Doble Grado de Ingeniería Civil e Ingeniería de los Recursos Mineros y Energéticos. Director del Proyecto: Agustin Menendez Díaz.

(cc) EY-NC-ND 2018, Universitat Politècnica de València

Congreso IN-RED (2018) 
Consideramos que se han cumplido con holgura los objetivos planteados, ya que en primer y segundo curso de las carreras en las que hemos desarrollado el proyecto de innovación docente, los alumnos aun ven lejano orientar su proyecto fin de grado

\section{Conclusiones y valoración de global del proyecto.}

El trabajo desarrollado nos permite contar con un fondo de proyectos ligados a edificios de nuestra universidad, perfectamente documentamos y que pueden usarse como ejemplos de aplicación en cursos posteriores.

Entre los puntos fuertes podemos indicar:

- Se ha fortalecido la formación multidisciplinar por el intercambio de profesores de varias titulaciones y departamentos, lo que permite a los alumnos ver los problemas en su conjunto, pero también con nuevas vertientes y ramificaciones técnicas.

- Los resultados de la evaluación y la encuesta de alumnos nos ha permite valorar dicha actividad de forma positiva, animándonos a trasladar para cursos posteriores estas actividades de innovación docente de una forma más regular, e insertarlas dentro de las actividades normales de un cuatrimestre.

- Por otra parte, creemos que este proyecto nos ha permitido mejorar la percepción que los alumnos tienen de los edificios de nuestra Universidad. Son capaces de identificar más elementos funcionales de los edificios, y son más conscientes de los problemas que pueden producirse si no se mantienen adecuadamente.

Entre los puntos débiles debemos indicar:

- Las limitaciones en tiempo no nos han permitieron debatir los proyectos de las obras de cada edificio, congregando en una misma sesión a alumnos de distintas asignaturas y titulaciones. Hubiese sido preciso planificar el traslado de alumnos de un edifico a otro, y coordinar horarios intentando afectar lo menos posible a otras asignaturas, de ahí que no se planteó su realización conjunta.

- Las obras visitadas corresponden obras realizadas la Universidad de Oviedo, pero hubiese sido interesante ver otros proyectos similares en edificios del entorno.

- Es difícil coordinar las visitas a obras e instalaciones en funcionamiento, especialmente cuando dichas obras se están realizando, o cuando las instalaciones están en pleno fase de trabajo. Hay que garantizar la seguridad de los alumnos e interferir lo menos posible en las obras en realización. Esto hace que algunas de las visitas que podrían ser muy interesantes, no se haya podido realizar.

Finalmente queremos indicar que proyectos de innovación como el aquí desarrollado pueden aportar a la propia Universidad y a sus alumnos un valor añadido importante, e implicarlos de forma más firme en aspectos tan importantes como son las medidas de ahorro energético y sostenibilidad los edificios. 


\section{Referencias}

CHOI, J.; SHIN, J.; KIM, M.; KIM, I. (2016). "Development of openBIM-based energy analysis software to improve the interoperability of energy performance assessment”, Automation In Construction, 72:52-64, DOI: 10.1016/j.autcon.2016.07.004.

HAN, KK.; GOLPARVAR-FARD, M. (2017). "Potential of big visual data and building information modeling for construction performance analytics: An exploratory study”, Automation In Construction, 73: 184-198, DOI: 10.1016/j.autcon.2016.11.004.

LIU, R.; HATIPKARASULU Y. (2014). "Introducing Building Information Modeling Course into a Newly DevelopedConstruction Program with Various Student Backgrounds”, in 121st Asee Annual Conference \& Exposition, Indianapolis, Paper ID \#9291: 1-9.

MCGOUGH, D.; AHMED A.; AND AUSTIN S. (2013). "Integration of BIM in higher education: case study of the adoption of bim into Coventry university’s department of civil engineering, architecture and building”, in Sustainable Building Conference, Coventry University, UK., 394-403.

PIKAS, E.; SACKS R.; AND HAZZAN O. (2017). "Building information modeling education for construction engineering and management”. II: Procedures and implementation case study. Journal Of Construction Engineering And Management, 139:1-11.

(cc) EY-NC-ND 2018, Universitat Politècnica de València 